14.03

\title{
Мальформации как нарушение фрактальной структуры кровеносной системы организма
}

\author{
(C) В. Антонов, П. Ефрремов
}

Санкт-Петербургский политехнический университет Петра Великого, 195251 Санкт-Петербург, Россия

e-mail: antonovvi@mail.ru

Поступило в Редакцию 21 января 2020 г.

В окончательной редакции 21 января 2020 r.

Принято к публикации 17 февраля 2020 г.

Описаны математические модели, в основу которых положено рассмотрение кровеносной системы организма как мультифрактального объекта. Приведено решение двух задач. Первая связана с нормальным состоянием системы жизнеобеспечения организма, а именно теплообмен в коже человека Основой модели служат уравнения гидродинамики и теплообмена. Приведены количественные результаты расчетов тепловых потоков в трех слоях дермы. Вторая - с нарушением фрактальности за счет наличия артериовенозной мальформации в сосудистой системе головного мозга. Моделирование кровотока при наличии мальформации выполнено с помощью программного продукта SolidWorks 2017 Flow Simulation. Приведены данные о скоростях и потоках крови в сосудах для различных случаев расположения мальформации.

Ключевые слова: кровеносная система, фракталы, мальформации, моделирование.

DOI: 10.21883/JTF.2020.09.49683.24-20

В настоящее время для исследования процессов кровообращения широко применяется математическое и аналоговое моделирование. Основой математических моделей в большинстве случаев являются уравнения гидродинамики [1-12]. К особенностям физической модели следует отнести замкнутость и разветвленность сердечно-сосудистой системы, а также эластичность сосудов.

Современный взгляд на строение сосудистой системы состоит в том, чтобы рассматривать ее как фрактальный или, точнее, мультифрактальный объект (рис. 1).

Фрактальный анализ включает в себя определение фрактальной размерности и других фрактальных характеристик объекта. Фракталы, связанные с геометрическими объектами, удовлетворяют двум критериям: самоподобие и дробная размерность.

Мультифрактал состоит из отдельных частей, каждая из которых обладает своим свойством самоподобия. Важным примером применения мультифракталов является анализ сигналов и поведения хаотических систем.

Рассмотрим две задачи. Первая связана с нормальным состоянием системы жизнеобеспечения организма. Вторая - с нарушением фрактальности.

Моделирование теплообмена в коже человека выполнено методом конечных разностей. На рис. 2 представлена схема ячейки сосудистой система дермы. Ее можно рассматривать как трехуровневый мультифрактал.

В математическую модель стационарной теплопередачи в коже человека включены артерии, вены, венулы и капилляры. С помощью модели проведен анализ изменения температуры в ткани при изменении скорости движения крови по большим артериям, „тромбозу“ одной или нескольких вен и т.д. В настоящей работе рассмотрен случай теплообмена в термонейтральной зоне с температурой окружающей среды $28.5^{\circ} \mathrm{C}$.

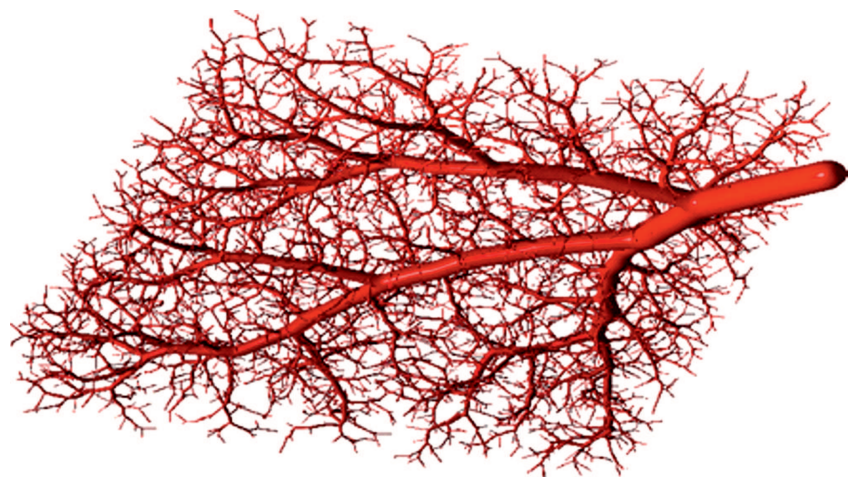

Рис. 1. Фрагмент сосудистой системы [18].

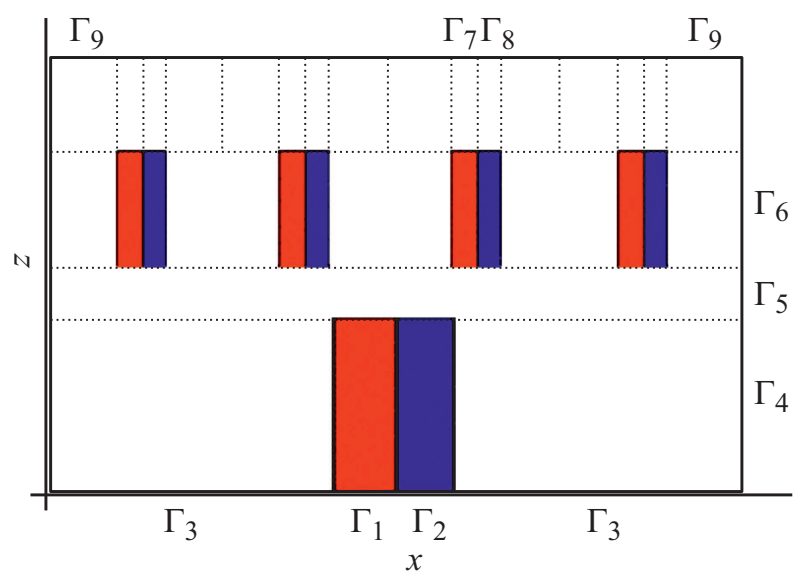

Рис. 2. Двумерная модель сосудистой системы дермы. 
Слои дермы состоят из сосудов и окружающей ткани. Ткани в дерме неоднородны, однако их теплопроводные свойства обычно не изменяются в пространстве. Это позволяет описать изменение температуры $\theta$ стационарным уравнением теплопроводности:

$$
-\lambda_{t} \nabla^{2} \theta=q^{v}
$$

Здесь $\lambda_{t}-$ теплопроводность ткани $\left[\mathrm{W} /\left(\mathrm{mm} \cdot{ }^{\circ} \mathrm{C}\right)\right]$, $q^{v}$ - удельная производительность внутренних источников тепла $\left[\mathrm{W} / \mathrm{mm}^{3}\right]$.

Кровь рассматривается как ньютоновская жидкость, течение которой можно описать приближением Буссинеска для несжимаемой жидкости

$$
\begin{gathered}
(\bar{v} \nabla) \bar{v}=-\frac{1}{\rho_{0}} \nabla P+v \nabla^{2} \bar{v}-g \beta\left(\theta-\theta_{0}\right), \\
(\bar{v} \nabla) \theta=a^{b l} \nabla^{2} \theta, \\
\operatorname{div} \bar{v}=0, \\
\rho=\rho_{0}\left[1-\beta\left(\theta-\theta_{0}\right)\right] .
\end{gathered}
$$

Здесь $\rho_{0}-$ плотность жидкости при температуре $\theta_{0}$,

$$
\beta=-\left.\frac{1}{\rho_{0}}\left(\frac{\partial \rho}{\partial \theta}\right)\right|_{\theta_{0}}
$$

- коэффициент температурного расширения, $v$ - кинематическая вязкость, $a$ - температуропроводность.

Граничные условия на границах $\Gamma_{1}-\Gamma_{9}$ поставлены традиционным способом. Например, на границе теплообмена с окружающей средой

$$
\mid \frac{\partial \theta}{\partial z}=-\frac{\alpha}{\lambda}\left(\theta-\theta_{c p}\right) .
$$

Геометрические параметры дермы и скорости кровотока приведены в табл. 1.

Полученная система уравнений приведена в безразмерную форму и построен разностный аналог методом контрольного объема. Зависимость температуры в модельных ячейках от скорости кровотока приведена в табл. 2.

Таблица 1. Параметры дермы и скорости кровотока

\begin{tabular}{l|c|c|c}
\hline \multicolumn{1}{c|}{ Величина } & Капилляры & $\begin{array}{c}\text { Второй } \\
\text { уровень }\end{array}$ & $\begin{array}{c}\text { Третий } \\
\text { уровень }\end{array}$ \\
\hline $\begin{array}{l}\text { Количество сосудов, } \\
\text { рiесеs/cm² }\end{array}$ & 6300 & 65 & 3 \\
Диаметр артерии, mm & 0.01 & 0.04 & 0.1 \\
Диаметр вены, mm & 0.01 & 0.1 & 0.25 \\
$\begin{array}{l}\text { Высота слоя, mm } \\
\text { Скорость кровотока }\end{array}$ & 2 & 3 & 4.5 \\
$\begin{array}{l}\text { в артерии, тm/s } \\
\text { Скорость кровотока }\end{array}$ & 1 & 8 & 20 \\
в вене, тm/s & & 2 & 2.3 \\
\end{tabular}

Таблица 2. Зависимость температуры в модельных ячейках от скорости кровотока

\begin{tabular}{c|c|c|c}
\hline $\begin{array}{c}\text { Скорость } \\
\text { кровотока, } \\
\mathrm{mm} / \mathrm{s}\end{array}$ & $\begin{array}{c}\text { Температура } \\
\text { на выходе из } \\
\text { артериолы, }{ }^{\circ} \mathrm{C}\end{array}$ & $\begin{array}{c}\text { Температура } \\
\text { в средней части } \\
\text { артериолы, }{ }^{\circ} \mathrm{C}\end{array}$ & $\begin{array}{c}\text { Температура } \\
\text { в средней части } \\
\text { вены, }{ }^{\circ} \mathrm{C}\end{array}$ \\
\hline 0 & 36.1097 & 36.2048 & 36.2048 \\
0.5 & 36.1098 & 36.2048 & 36.2048 \\
1 & 36.1098 & 36.2049 & 36.2048 \\
10 & 36.1104 & 36.2051 & 36.2046 \\
20 & 36.1112 & 36.2055 & 36.2044 \\
30 & 36.1121 & 36.2058 & 36.2041 \\
50 & 36.1097 & 36.2066 & 36.2035 \\
100 & 36.1181 & 36.2076 & 36.2026
\end{tabular}

Артериовенозная мальформация, которую можно рассматривать как нарушение фрактальности сосудистой системы головного мозга (ABM), представляет собой клубок аномальных кровеносных сосудов, соединяющих артерии и вены (рис. 3).

Люди могут родиться с АBM, но нарушения могут образовываться позже в жизни. Симптомы АВМ могут включать приступы, головную боль, мышечную слабость, потерю зрения, нарушение речи. Признаки АВМ обычно появляются в возрасте $10-40$ лет.

Существенным вкладом в аналоговое моделирование мальформаций явилась работа Е. Гао и соавторов [13]. Модель состоит из 55 артерий, 37 вен и 20 микроваскулярных групп. АВМ моделирована афферентными сосудами и дренирующими венами. Приведена зависимость давления от индекса кровотока $B P_{a}=71 \times$ $\times \exp \left(-1.47 \frac{F V I}{1000}\right)$, где: $F V I=0.25 D^{2} V \cdot 60(\mathrm{ml} / \mathrm{min})-$ индекс кровотока через афферентный сосуд, $D$ диаметр сосуда $[\mathrm{cm}], V-$ средняя скорость кровотока $[\mathrm{cm} / \mathrm{s}]$.

Нашей группой разработана модель АВМ на базе SolidWorks Flow Simulation. Мальформация представлена в виде посторонней связи между веной и артерией. Приняты следующие допущения: не рассматривается эластика сосудов, движение крови стационарно, известны данные о входных потоках. Для численной реализации модели приняты следующие параметры: диаметр артерии равен $0.5 \mathrm{~mm}$, диаметр вены равен $1.5 \mathrm{~mm}$, скорость кровотока на входе в артерию составляет $500 \mathrm{~mm} / \mathrm{s}$, что соответствует потоку жидкости $Q=V A=500 \pi r^{2}=98,125 \mathrm{~mm}^{3} / \mathrm{s}$. Давление в сосудах мозга равно $5332.9 \mathrm{~Pa}$. Распределение скоростей в артерии, вене и мальформации приведены на рис. 4.

Подробное изложение состояния дел по диагностике и лечению мальформаций можно найти в [14-17]. Современными методами лечения мальформаций являются эмболизация и гамма-нож. Эмболизация - рентгенохирургическая эндоваскулярная процедура, которая состоит в избирательной окклюзии (закупорке) кровеносных сосудов специально введенными эмболами. 


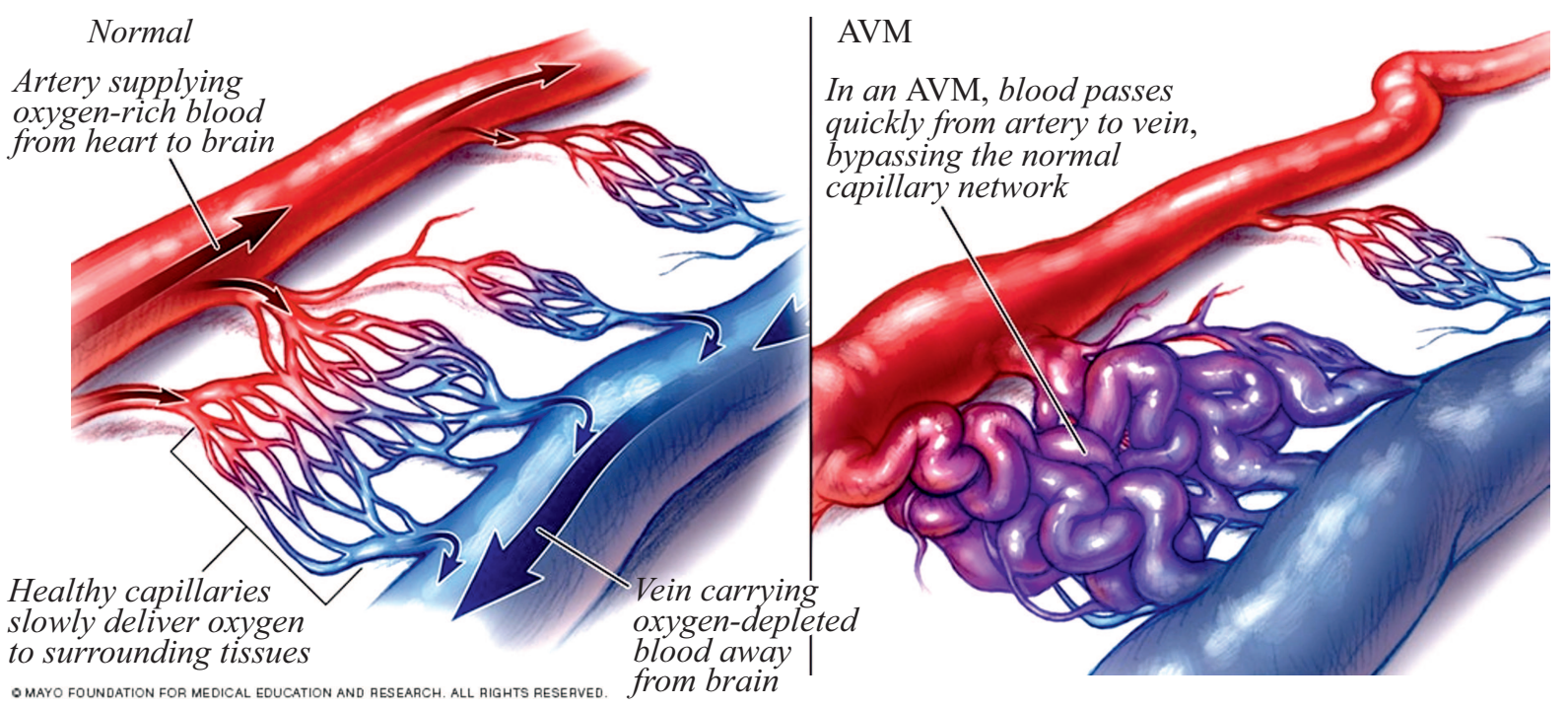

Рис. 3. Артериовенозная мальформация [19].

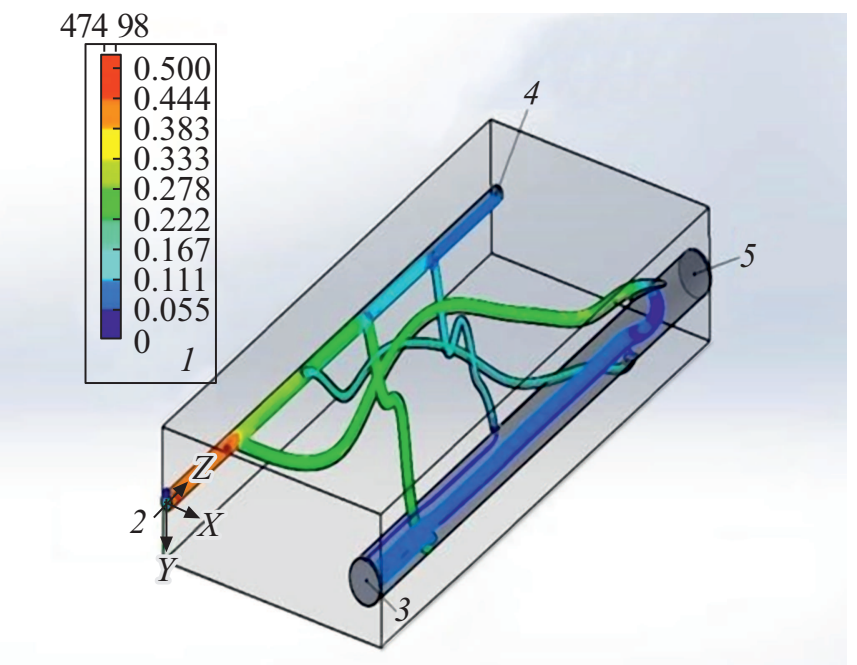

Рис. 4. Распределение скоростей в мальформации: 1 - скорость кровотока $[\mathrm{m} / \mathrm{s}], 2-$ мозговой кровоток $600 \mathrm{ml} / \mathrm{min}, \quad 3$ - давление $5332 \mathrm{~Pa}, 4$ - артериальный кровоток $180 \mathrm{ml} / \mathrm{min}, 5$ - венозный кровоток $180 \mathrm{ml} / \mathrm{min}$.

Гамма-нож использует ионизирующее излучение, источником для которого является Кобальт-60. По сравнению с радиохирургией, гамма-нож имеет большую пространственную точность (свыше $0.5 \mathrm{~mm}$ ) и меньшую равномерность дозы внутри мишени (наиболее распространено облучение 50\% изодозой), тогда как на линейных ускорителях используются изодозы 80-90\% [17].

Bblводы. Современный подход к описанию структур человеческого организма содержит элементы мультифрактального анализа. Такой подход позволяет выявить наиболее характерные особенности, присущие системе регуляции большинства физиологических процессов. На- рушение фрактальности в строении и поведении системы жизнеобеспечения может служить первым признаком начала болезни или даже приближения критической ситуации. Математические модели, в основе которых лежит анализ хаотического поведения динамических систем, дают медикам дополнительную информацию для принятия решений о выборе пути лечения пациентов.

\section{Благодарности}

Авторы выражают благодарность руководителю лаборатории церебральной патологии Нейрохирургического института Поленова В.Б. Семенютину за предоставленную информацию.

\section{Вклад авторов}

Антонов В.И. - Концепция исследования, теплообмен в коже; Ефремов П. - Модель мальформации.

\section{Конфликт интересов}

Авторы заявляют, что у них нет конфликта интересов.

\section{Список литературы}

[1] Холодов А.С. // Компьютерные модели и прогресс медицины. / Под ред. Белоцерковского О.М., Холодова А.С. М.: Наука, 2001. С. 127-163.

[2] Холодов А.С., Симаков С.С. // Математическое моделирование. 2008. Т. 20 (4). С. 87-102.

[3] Kholodov A.S., Simakov S.S. // J. Biomechanics. 2006. Vol. 39. Supplement 1. P. 401.

[4] Ursino M., Lodi C.A. // J. Appl. Physiol. 1997. Vol. 82. N 4. P. 1256-1269. 
[5] Баранов А.П., Клименок М.Ф. Медицинская и биологическая физика. Витебский гос. мед. ун-т. 2-е изд. Витебск : ВГМУ, 2010. $390 \mathrm{c}$.

[6] Семенов Ю.С., Дьяченко А.И. // Труды МФТИ. 2014. Т. 6. № 3. C. $102-113$.

[7] Петров И.Б. // Труды МФТИ. 2009. Т. 1. № 1. С. 5-14.

[8] Gosfa K.D., Hunter P.J., Pogers J.M., Gussione G.M., Waldmen L.K. // Part I. ASME J. Biomech. Eng. 1996. N 118 (4). P. 452-463.

[9] Ашметов И.В., Буничева А.Я., Мухин С.И., Соколова Т.В., Соснин Н.В., Фаворский А.П. // Компьютер и мозг. Новые технологии. М.: Наука, 2005. С. 321-337.

[10] Gataulin Y.A., Zaitsev D.K., Smirnov E.M., Yukhnev A.D. // Rus. J. Biomechan. 2019. Vol. 23. N 1. P. 58-66.

DOI: 10.15593R.J. Biomech.,2019.1.07

[11] Gataulin Y.A., Yukhnev A.D., Zaitsev D.K., Smirnov E.M., Kulikov V.P., Kirsanov R.I. // J. Physics: Conf. Series. 2018. Vol. 1135. P. 012089. (7 p).

DOI: $10.1088 / 1742-6596 / 1135 / 1 / 012089$

[12] Gataulin Y.A., Zaitsev D.K., Smirnov E.M., Yukhnev A.D. // J. Phys. Mathem. 2017. Vol. 3. N 1. P. 1-6. https:doi.org/10.1016/j.spjpm.2017.02.001

[13] Young W.L., Gao T., Hademenos G.J., Massoud T.F. // Intracranial Arteriovenous Malformation. Informa Healthcare. USA. 1997. P. 49-70.

[14] Spetzler R.F., Wilson C.B., Weinstein P., Mehdorn M., Townsend J., Telles D. Normal Perfusion Pressure Breakthrough Theory. Clin Neurosurg. 1978. Vol. 25. P. 651672.

[15] Quick C.M., Hashimoto T., Young W.L. // Neurol. Res. 2001. Vol. 23. P. 641-644.

[16] Панунцев В.С., Воронов В.Г., Никитин П.И., Алиев В.А., Байрамов Р.Р., Бухаев И.М., Гафуров Р.Р., Дрягина Н.В., Панунщев Г.К., Раджабов С.Д., Размологова О.Ю., Рожченко Л.В., Семенютин В.Б. Современные представления о церебральных артериовенозных мальформациях СПб.: Синтез Бук, 2013. 427 c.

[17] Mack A., Heinz Czempiel, Hans-Jürg Kreiner, Gerhard Dür, Berndt Wowra // Medical Phys. 2002. Vol. 29. N 561. P. 561-568. https://doi.org/10.1118/1.1463062

[18] Taringa.net. Espinacos convertidas en celulas del Corazon.

[19] Электронный pecypc. Mayoclinic.org. 